\title{
Mid-Infrared Pulsed Laser Lithotripsy with a Tunable Laser Using Difference-Frequency Generation
}

\author{
Hisanao Hazama ${ }^{1}$, Hiromu Kutsumi ${ }^{2}$, Kunio Awazu ${ }^{1,3,4}$ \\ ${ }^{1}$ Graduate School of Engineering, Osaka University, Osaka, Japan \\ ${ }^{2}$ Graduate School of Medicine, Kobe University, Kobe, Japan \\ ${ }^{3}$ Graduate School of Frontier Biosciences, Osaka University, Osaka, Japan \\ ${ }^{4}$ The Center for Advanced Medical Engineering and Informatics, Osaka University, Osaka, Japan \\ Email: hazama@wakate.frc.eng.osaka-u.ac.jp
}

Received May 1, 2013; revised June 20, 2013; accepted July 29, 2013

Copyright (c) 2013 Hisanao Hazama et al. This is an open access article distributed under the Creative Commons Attribution License, which permits unrestricted use, distribution, and reproduction in any medium, provided the original work is properly cited.

\begin{abstract}
A novel technique of lithotripsy was investigated with a mid-infrared tunable pulsed laser using difference-frequency generation (DFG). Human gallstone samples obtained from 24 patients were analyzed with their infrared absorption spectra. It was found that the principal components of the gallstones were different for the different patients and that the gallstone samples used in this research could be classified into four groups, i.e., mixed stones, calcium bilirubinate stones, cholesterol stones, and calcium carbonate stones. In addition, some gallstone samples had different compositions within the single stone. The mid-infrared laser tunable within a wavelength range of $5.5-10 \mu \mathrm{m}$ was irradiated to the cholesterol stones at two different wavelengths of 6.83 and $6.03 \mu \mathrm{m}$, where the cholesterol stones had relatively strong and weak absorption peaks, respectively. As the result, the cholesterol stones were more efficiently ablated at the wavelength of $6.83 \mu \mathrm{m}$ with the strong absorption peak. Therefore, it is suggested that the gallstones could be efficiently ablated by tuning the wavelength of the laser to the strong absorption peak of the gallstones. The higher efficiency of the ablation using the characteristic absorption peaks should lead to the safer treatment without damage to the surrounding normal tissues. In order to identify the composition of the gallstones in the patients, endoscopic and spectroscopic diagnosis using the DFG laser and an optical fiber probe made with two hollow optical fibers and a diamond attenuation total reflection prism should be useful. The absorption spectrum of the gallstones in the patients could be measured by measuring the energy of the DFG laser transmitted through the optical fiber probe and by scanning the wavelength of the DFG laser.
\end{abstract}

Keywords: Gallstone; Lithotripsy; Mid-Infrared Tunable Laser; Difference-Frequency Generation

\section{Introduction}

Recently, the cholelithiasis has been the most popular in the diseases of a bile duct or a gallbladder, and it has been said that one of ten people has gallstones [1]. They basically have no symptom, and about $80 \%$ of them have no problem in their lives. These stones are called "silent stones". If the stone is small, it is discharged to small intestine through the bile duct in safety or stayed in the bile duct without feeling pain. However, when a gallstone gets larger, it clogs up the duct and starts to feel a lot of pain involved with nausea and vomit. In addition, there is no denying that bacteria are growing and the infection of bile duct or sometimes hepatic abscess develops. As the symptom progresses, the patient also gets fever, chill, and jaundice. If the worst happens, the patient develops the infection disease which has a risk of death called "bacterial cholangitis". The bacterial cholangitis gives the patient a risk of death because bacteria infect various parts of whole body through the blood flow. The risk factors of gallstone formation are age, fatness, western diet, overstress, and so on. The number of the patients of gallstones has increased, so it is important to establish the more effective and less invasive treatment as soon as possible.

Gallstones can be roughly classified into cholesterol stone, dye stone, and other stones by their components. Cholesterol stone can be more classified into pure cholesterol stone, mixed stone, and combined stone. On the other hand, dye stone can be also more classified into calcium bilirubinate stone, and black stone. Other stones contain calcium carbonate stone and aliphatic acid calcium stone. 
In addition, gallstones can be categorized into gallbladder stones, stones in the bile duct, and intrahepatic bile duct stones by location of developing gallstones. Gallbladder stones are developed in gallbladder, and most of them are cholesterol stones and black stones. Stone in the bile duct is developed in the bile duct, and greater parts of them are swept away by bile released from gallbladder. Intrahepatic bile stones are developed in hepatic bile duct, and they are rarely developed.

The treatment of gallbladder stones is generally performed with laparoscopical cholecystectomy. This is the operation that a doctor inserts a tube from a small incised hole of abdominal area and extracts the gallbladder filled with gallstones through the hole. This treatment is used all over the world because the cholelithiasis is completely cured with a relatively easy procedure.

On the other hand, stones in the bile duct and intrahepatic bile duct stones had been treated with surgical procedures before. Then, endoscopic retrograde cholangio pancreatography (ERCP) has been developed in 1969, and other medical managements such as endoscopic sphincterotomy (EST), electrohydraulic lithotripsy (EHL), and extracorporeal shock wave lithotripsy (ESWL) have been developed. These therapies enabled doctors to eliminate gallstones without incising abdominal areas and its success rate is just over or below $90 \%$, so the quality of life (QOL) of patients is greatly improved. However, the therapies also had problems of an antidromic infection, recurrence of gallstones, and so on. In addition, some stones called "difficult-to-treat-stones" cannot be eliminated because of their hardness, hugeness, place of emergence, and the rest. Besides, the difficulty of the usage prevents these therapies from being popular, so it is necessary for us to develop more effective treatment.

Since the initial introduction of the continuous wave $\mathrm{CO}_{2}$ laser (wavelength $\lambda=10.6 \mu \mathrm{m}$ ) for fragmentation of human gallstones, several laser lithotriptors, including Ho:YAG $(\lambda=2.08 \mu \mathrm{m}), Q$-switched Nd:YAG $(\lambda=1.06$ $\mu \mathrm{m})$, and pulsed dye $(\lambda=504 \mathrm{~nm})$ lasers have been investigated and clinically used [2]. Especially, the Ho: YAG laser is an attractive alternative to other conventional laser lithotriptors because it offers high power pulses ( $\sim \mathrm{kW}$, up to several joules at $250 \mu$ pulse duration) with higher transmittance than pulsed-dye laser systems [3]. However, the accidental irradiation to surrounding normal tissues can lead to the damage to the normal tissue, and the recurrence rate of the stones are not negligible. Therefore, these laser lithotriptors have not widely been used in the clinical treatment [4].

In this paper, we discuss the feasibility of mid-infrared laser lithotripsy with a goal of elimination of gallstones in the bile duct. We investigated the gallstone fragmentation with a mid-infrared tunable pulsed laser using difference-frequency generation as their alternative light source. The irradiation of the mid-infrared laser induces vibration in the unique intramolecular bond of a gallstone, and the low-power lithotripsy is expected to be possible. Therefore, we can minimize the risk of damage to the normal tissue surrounding the gallstones, and the procedure could easily be completed in a shorter time.

\section{Materials and Methods}

\subsection{Gallstone Samples and Measurement of Their Infrared Absorption Spectra}

Human gallstone samples were obtained from 24 patients of the Kobe University Hospital in Japan. Figure 1 shows photographs of the gallstone samples used in this research. Their chemical compositions were analyzed with a Fourier transform infrared spectrometer (FT-IR) using potassium bromide $(\mathrm{KBr})$ pellet method [5]. To make the sample pellets, $\mathrm{KBr}$ and each gallstone sample were ground together in a mortar. Then the powder of the mixture of $\mathrm{KBr}$ and the gallstone sample was pressed in a cell. The pellet was formed by application of adequate pressure for several seconds. The concentration of the gallstone sample in $\mathrm{KBr}$ was optimized to obtain sufficient signal-to-noise ratio in the absorption spectrum measured with the FT-IR. Some gallstone samples (sample number 2, 3, 4, 7, 17, 18, 19, and 20) showed different color for inner and outer parts within the single stone. Therefore, the absorption spectra were separately measured for inner and outer parts of these gallstone samples.

\subsection{Irradiation of the Mid-Infrared Laser to the Gallstone Samples}

The gallstones were polished with a polishing machine to obtain a flat surface and stored in distilled water for an hour.

A mid-infrared tunable pulsed laser developed by Kawasaki Heavy Industries, Ltd. (Hyogo, Japan) and RIKEN (Saitama, Japan) was used [6,7]. Figure 2 shows the schematic of the mid-infrared tunable laser using difference-frequency generation (DFG) between a $Q$ switched Nd:YAG laser (Tempest 10, New Wave Research, Inc., CA, USA) with a wavelength of $1064 \mathrm{~nm}$ and a Cr:forsterite laser tunable within a wavelength range of 1180 - $1350 \mathrm{~nm}$ pumped by another $Q$-switched Nd:YAG laser (INDI-40-10, Spectra Physics Inc., CA, USA). The DFG output was obtained using two silver gallium sulfide $\left(\mathrm{AgGaS}_{2}\right)$ crystals. The mid-infrared laser was tunable within a wavelength range of $5.5-10.0$ $\mu \mathrm{m}$ with a step of $0.01 \mu \mathrm{m}$, and the wavelength tuning procedure was automatically controlled with a software running on a personal computer. The maximum pulse energy of the mid-infrared laser was about $1.4 \mathrm{~mJ}$ at the wavelength of $5.5 \mu \mathrm{m}$. The mid-infrared laser had a pulse width of $5 \mathrm{~ns}$ and a pulse repetition rate of $10 \mathrm{~Hz}$. 


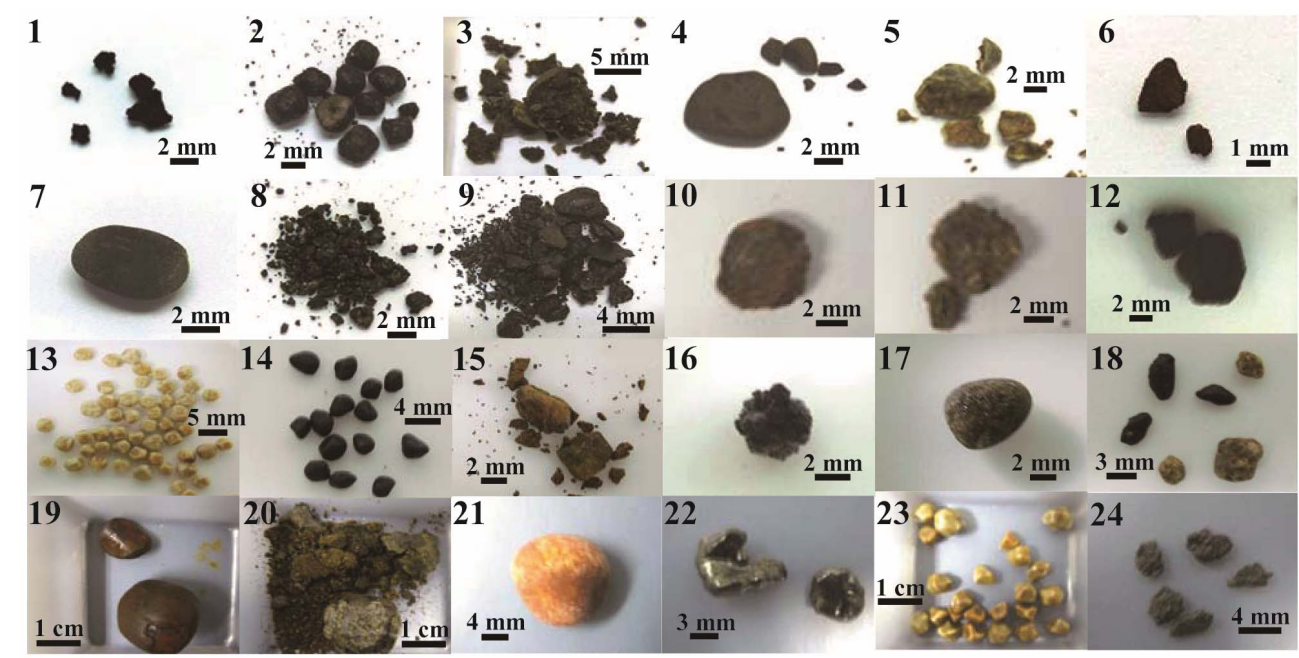

Figure 1. Photographs of the human gallstone samples used in this research.

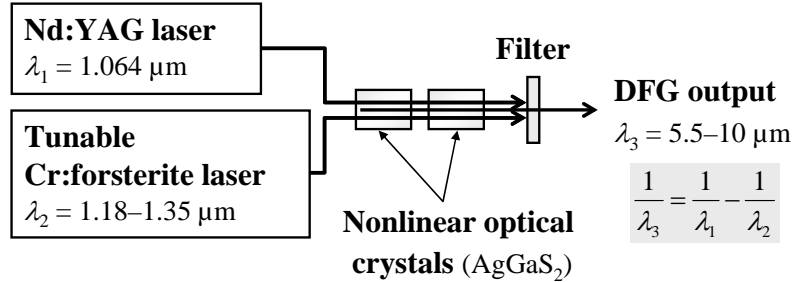

Figure 2. Schematic of the mid-infrared tunable laser using difference-frequency generation.

The mid-infrared laser beam was delivered to the gallstone sample using gold coated flat mirrors and focused onto the surface of the gallstone using a zinc selenide (ZnSe) lens with a focal length of $51 \mathrm{~mm}$. The spatial beam profile of the focus on the sample surface was measured using knife-edge method. A knife-edge was scanned across the laser beam at the focal point with a constant pitch of $20 \mu \mathrm{m}$. The transmitted laser energy past the knife-edge was measured using an energy meter equipped with a pyroelectric sensor (PE10, OPHIR Optronics, Israel). As the result, the focus diameter (full width at the half maximum) was estimated as $140 \mu \mathrm{m}$.

The surface of the gallstone during laser irradiation was observed with a charge-coupled device (CCD) camera equipped with an optical microscope (129406, KEYENCE Corp., Japan). After the laser irradiation, the gallstone samples were dried and the area and depth of the ablated crater were measured using a confocal laser scanning microscope (VK7510, KEYENCE Corp.).

\section{Results and Discussion}

\subsection{Infrared Absorption Spectra of the Gallstone Samples}

Figure 3 shows typical infrared absorption spectra of the human gallstone samples shown in Figure 1. It was found that the absorption spectra of the gallstone samples were different for the different patients and that the gallstone samples with different colors for inner and outer parts (sample number 2, 3, 4, 7, 17, 18, 19, and 20) had different absorption spectra within the single stone. It was also found that the absorption spectra of the gallstone samples used in this research could be classified into four types as shown in Figure 3. From the characteristic peaks in these absorption spectra, it was supposed that the four absorption spectra shown in Figure 3 were corresponding to the mixed stones, calcium bilirubinate stones, cholesterol stones, and calcium carbonate stones, respectively [8]. The results of the classification of the gallstone samples are summarized in Table 1.

\subsection{Dependence of the Irradiation Effects on the Laser Wavelength}

In the laser irradiation experiments, cholesterol stones (sample number 13) were used. To evaluate the effect of the laser wavelength, the DFG laser was irradiated at two different wavelengths, i.e., $6.03 \mu \mathrm{m}$ with relatively weak absorption peak and $6.83 \mu \mathrm{m}$ with relatively high absorption peak as shown in Figure 3(c). The average power density was varied within a range of $2-52 \mathrm{~W} / \mathrm{cm}^{2}$. The photomicrographs of the ablated craters in Figure 4 illustrate a visual comparison of the difference for each wavelength and each power density of the DFG laser irradiation. Figures 4(a)-(e) show the craters with the wavelength of $6.83 \mu \mathrm{m}$, and Figures $\mathbf{4 ( f ) - ( j )}$ show them with the wavelength of $6.03 \mu \mathrm{m}$. As the power density was increased, the size of the crater became larger for both wavelengths. However, the photomicrographs show the craters were produced with lower power density for the wavelength of $6.83 \mu \mathrm{m}$ with the strong absorption peak. On the other hand, Figure 5 shows the area and depth of the ablated crater, respectively, as a function of 


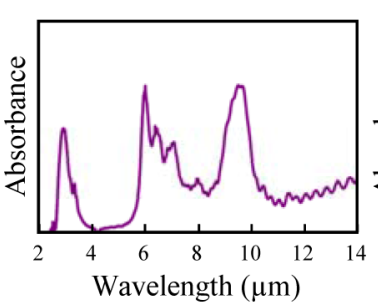

(a)

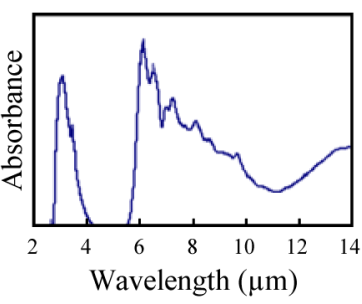

(b)

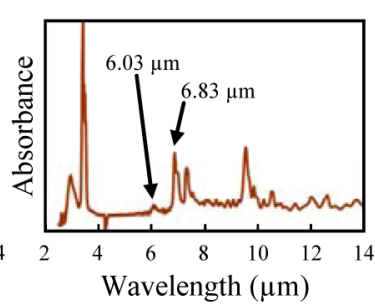

(c)

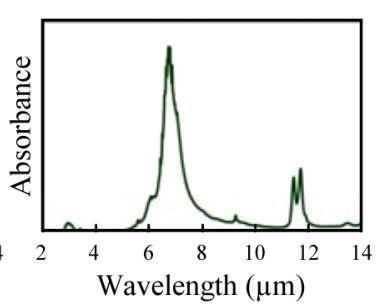

(d)

Figure 3. Typical infrared absorption spectra of the human gallstone samples.

Table 1. Classification results of the human gallstone samples using infrared absorption spectra. A: mixed stone; B: calcium bilirubinate stone; $C$ : cholesterol stone; and D: calcium carbonate stone.

\begin{tabular}{|c|c|c|c|c|c|c|c|c|c|c|c|c|}
\hline Sample number & 1 & 2 & 3 & 4 & 5 & 6 & 7 & 8 & 9 & 10 & 11 & 12 \\
\hline Inner part & \multirow[b]{2}{*}{ B } & $\mathrm{C}$ & A & A & \multirow[b]{2}{*}{ C } & \multirow[b]{2}{*}{ B } & B & \multirow[b]{2}{*}{ B } & \multirow[b]{2}{*}{ B } & \multirow[b]{2}{*}{$\mathrm{D}$} & \multirow[b]{2}{*}{$\mathrm{D}$} & \multirow[b]{2}{*}{ B } \\
\hline Outer part & & $\mathrm{B}$ & $\mathrm{C}$ & $\mathrm{C}$ & & & D & & & & & \\
\hline Sample number & 13 & 14 & 15 & 16 & 17 & 18 & 19 & 20 & 21 & 22 & 23 & 24 \\
\hline $\begin{array}{l}\text { Inner part } \\
\text { Outer part }\end{array}$ & $\mathrm{C}$ & B & B & B & $\begin{array}{l}\text { D } \\
\text { C }\end{array}$ & $\begin{array}{l}\mathrm{C} \\
\mathrm{A}\end{array}$ & $\begin{array}{l}\text { A } \\
\text { B }\end{array}$ & $\begin{array}{l}\mathrm{D} \\
\mathrm{B}\end{array}$ & C & A & $\mathrm{C}$ & B \\
\hline
\end{tabular}

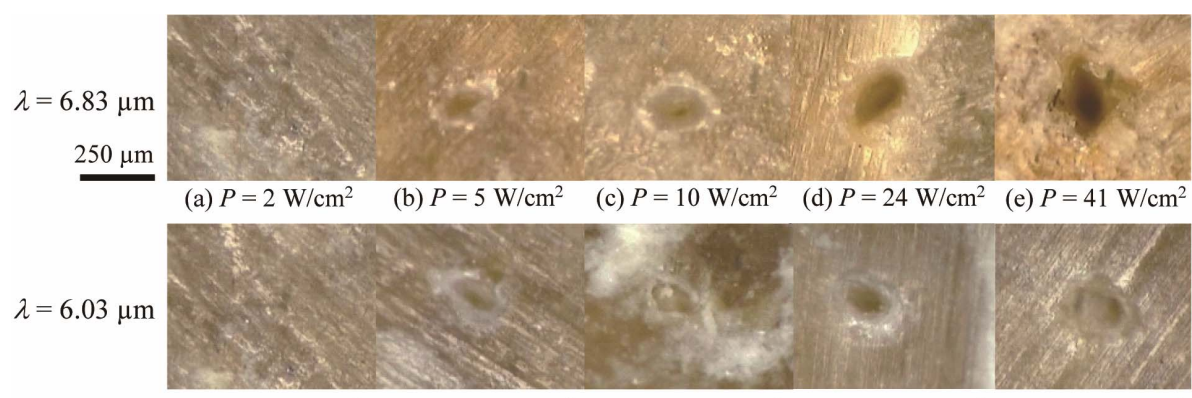

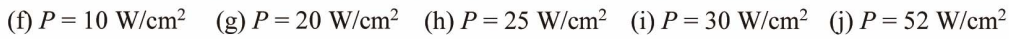

Figure 4. Photomicrographs of the human gallstones after the irradiation of the DFG laser, where $\lambda$ and $P$ denote the wavelength and average power density of the laser, respectively.

the average power density at the laser wavelengths of 6.83 and $6.03 \mu \mathrm{m}$. It was found that the threshold power density of the ablation was lower for the wavelength of $6.83 \mu \mathrm{m}$ and that both the area and depth of the crater produced by the laser irradiation at the wavelength of $6.83 \mu \mathrm{m}$ were larger and deeper than those produced at the wavelength of $6.03 \mu \mathrm{m}$ within the average power density of $0-20 \mathrm{~W} / \mathrm{cm}^{2}$. On the other hand, with the power density higher than $20 \mathrm{~W} / \mathrm{cm}^{2}$, the area and depth of the craters produced with the wavelength of $6.83 \mu \mathrm{m}$ were similar to them produced with the wavelength of $6.03 \mu \mathrm{m}$. These results at the average power density higher than $20 \mathrm{~W} / \mathrm{cm}^{2}$ should be caused by our experimental setup with the fixed focal point during the laser irradiation. The higher efficiency of the ablation with the wavelength of $6.83 \mu \mathrm{m}$ is due to the higher absorption coefficient of the cholesterol stones. The higher efficiency of the ablation using the characteristic absorption peaks should lead to the safer treatment without damage to the surrounding normal tissues. In order to identify the composition of the gallstones in the patients, endoscopic and spectroscopic diagnosis using the DFG laser and an optical fiber probe made with two hollow optical fibers [9-11] and a diamond attenuation total reflection (ATR) prism should be useful [7]. The absorption spectrum of the gallstones in the patients could be measured by measuring the energy of the DFG laser transmitted through the optical fiber probe and by scanning the wavelength of the DFG laser.

\section{Conclusion}

A novel technique of lithotripsy was investigated using a mid-infrared pulsed laser tunable within a wavelength range of 5.5 - $10 \mu \mathrm{m}$. Human gallstone samples obtained from 24 patients were analyzed with their infrared absorption spectra. It was found that the principal components of the gallstones were different for the different 


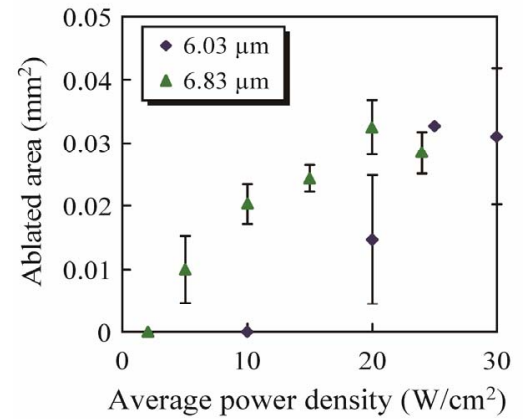

(a)

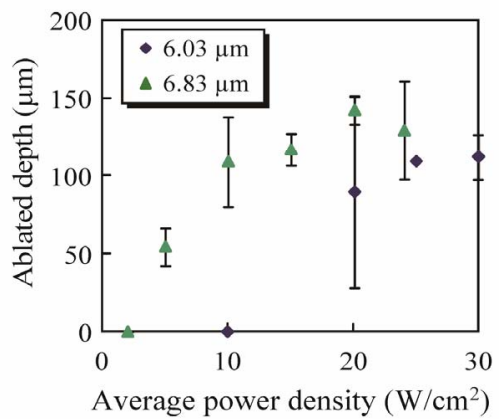

(b)

Figure 5. Ablated crater area and depth produced by the irradiation of the DFG laser at the wavelengths of 6.83 and $6.03 \mu \mathrm{m}$.

patients and that the gallstone samples used in this research could be classified into four groups, i.e., mixed stones, calcium bilirubinate stones, cholesterol stones, and calcium carbonate stones. In addition, some gallstone samples had different compositions within the single stone. The mid-infrared laser using DFG was irradiated to the cholesterol stones at two different wavelengths of 6.83 and $6.03 \mu \mathrm{m}$, where the cholesterol stones had relatively strong and weak absorption peaks, respectively. As the result, the cholesterol stones were more efficiently ablated at the wavelength of $6.83 \mu \mathrm{m}$ with the strong absorption peak. Therefore, it is suggested that the gallstones could be efficiently ablated by tuning the wavelength of the laser to the strong absorption peak of the gallstones. The higher efficiency of the ablation using the characteristic absorption peaks should lead to the safer treatment without damage to the surrounding normal tissues. The endoscopic and spectroscopic diagnosis using the DFG laser and the optical fiber probe made with the hollow optical fibers and the diamond ATR prism should be useful for the endoscopic analysis of the composition of the gallstones in the patients.

\section{Acknowledgements}

We thank Shinya Yamada and Masahiro Tsukamoto of Osaka University and Minoru Yokoyama of Kawasaki Heavy Industries, Ltd. for their technical supports. This work was partially supported by Hyogo COE Program Promotion Project.

\section{REFERENCES}

[1] P. J. Blind and M. Lundmark, "Management of Bile Duct Stones: Lithotripsy by Laser, Electrohydraulic, and Ultrasonic Techniques,” European Journal of Surgery, Vol. 164, No. 6, 1998, pp. 403-409. doi:10.1080/110241598750004201

[2] H. Lee, H. W. Kang, J. M. H. Teichman, J. Oh and A. J. Welch, "Urinary Calculus Fragmentation during Ho:YAG and Er:YAG Lithotripsy," Lasers in Surgery and Medicine, Vo. 38, No. 1, 2006, pp. 39-51.

\section{doi:10.1002/lsm.20258}

[3] K. F. Chan, G. J. Vassar, T. J. Pfefer, J. M. H. Teichman, R. D. Glickman, S. T. Weintraub and A. J. Welch, "Holmium:YAG Laser Lithotripsy: A Dominant Photothermal Ablative Mechanism with Chemical Decomposition of Urinary Calculi,” Lasers in Surgery and Medicine, Vol. 25, No. 1, 1999, pp. 22-37. doi:10.1002/(SICI)1096-9101(1999)25:1<22::AID-LSM4 $>3.0 . \mathrm{CO} ; 2-6$

[4] M. Watanabe, H. Kajiwara, K. Awazu and K. Aizawa, "Bilirubin Caluculi Crushing by Laser Irradiation at a Molecular Oscillating Region Wavelength Based on Infrared Absorption Spectrum Analysis Using a Free-Electron Laser,” Surgery Today, Vol. 31, No. 7, 2001, pp. 626-633. doi:10.1007/s005950170097

[5] K. Iwai, Y. W. Shi, Y. Matsuura, M. Miyagi, S. Saito and Y. Arai, "Characteristics of Calculus Fragmentation with Er:YAG Laser Light Emitted by an Infrared Hollow Optical Fiber with Various Sealing Caps," Applied Optics, Vol. 44, No. 16, 2005, pp. 3266-3270.

doi:10.1364/AO.44.003266

[6] H. Hazama, Y. Takatani and K. Awazu, "Integrated Ultraviolet and Tunable Mid-Infrared Laser Source for Analyses of Proteins,” Proceedings of SPIE, Vol. 6455, 2007, Article ID: 645507. doi:10.1117/12.700112

[7] H. Hazama, K. Ishii and K. Awazu, "Less-Invasive Laser Therapy and Diagnosis Using a Tabletop Mid-Infrared Tunable Laser," Journal of Innovative Optical Health Sciences, Vol. 3, No. 4, 2010, pp. 285-292. doi:10.1142/S179354581000109X

[8] E. Laloum, N. Q. Dao and M. Daudon, “Cluster Analysis of Gallstone FT-IR Spectra: Tests on Simulated Mixture Spectra and Comparison between Spectral and Morphological Classification of Human Gallstones," Applied Spectroscopy, Vol. 52, No. 9, 1998, pp. 1210-1221. doi:10.1366/0003702981945039

[9] J. A. Harrington, "Infrared Fibers and Their Applications,” SPIE Press, Washington, 2004. doi:10.1117/3.540899

[10] Y. W. Shi, Y. Wang, Y. Abe, Y. Matsuura, M. Miyagi, S. Sato, M. Taniwaki and H. Uyama, "Cyclic Olefin Polymer-Coated Silver Hollow Glass Waveguides for the Infrared," Applied Optics, Vol. 37, No. 33, 1998, pp. 77587762. doi:10.1364/AO.37.007758 
[11] Y. W. Shi, K. Ito, Y. Matsuura and M. Miyagi, "Multiwavelength Laser Light Transmission of Hollow Optical Fiber from the Visible to the Mid-Infrared," Optics Let- ters, Vol. 30, No. 21, 2005, pp. 2867-2869.

doi:10.1364/OL.30.002867 